\title{
Methylation of the miR-29b-3p promoter contributes to angiogenesis, invasion, and migration in pancreatic cancer
}

\author{
LIHUA WANG $^{1 *}, \mathrm{NV} \mathrm{MU}^{2 *}$ and NINA QU ${ }^{3}$ \\ Departments of ${ }^{1}$ Gastroenterology, ${ }^{2}$ Outpatient Medicine and ${ }^{3}$ Ultrasound, Yantai Yuhuangding \\ Hospital of Qingdao University, Yantai, Shandong 264000, P.R. China
}

Received December 5, 2019; Accepted July 2, 2020

DOI: $10.3892 /$ or.2020.7832

\begin{abstract}
The aim of the present study was to investigate the effects of microRNA (miR)-29b-3p gene promoter methylation on angiogenesis, invasion, and migration in human pancreatic cancer. Prediction of promoter methylation of miR-29b-3p was performed through the MethPrimer tool. Then the methylation levels of miR-29b-3p in human pancreatic cancer tissues and cell lines were detected by pyrosequencing, and the relative expression of miR-29b-3p was assessed in pancreatic cancer tissues by qPCR. The results were analyzed by linear regression. Western blot analysis was used to detect expression of DNA methyltransferases (DNMTs) in pancreatic cancer tissues and adjacent tissues. The Transwell assay was used to detect the ability of cell migration and invasion. Cells were co-cultured with human umbilical vein endothelial cells (HUVECs) to detect the ability of angiogenesis. The results revealed that DNMT1 expression in pancreatic cancer tissues was higher than that in adjacent tissues. Further results showed that expression of miR-29b was negatively correlated with the methylation level of the miR-29b promoter. Bxpc3 and Capan-2 cells had higher methylation levels, and the expression level of miR-29b-3p in Bxpc3 and Capan-2 cells was found to be lower than that of other cell lines. Expression of zonula occludens-1 (ZO-1) and occludin was significantly increased, and the migration of cancer cells was decreased after cells were treated with siRNA DNMT1. Further results showed that miR-29b reversed the promotive effect of DNMT1 overexpression on tumor cell malignant properties. Methylation of the miR-29b-3p promoter contributes to angiogenesis, invasion, and migration in pancreatic cancer. This study indicated that the alteration of methylation of mR-19b may be a potential approach for inhibiting the progression of pancreatic cancer.
\end{abstract}

Correspondence to: Dr Nina Qu, Department of Ultrasound, Yantai Yuhuangding Hospital of Qingdao University, 20 Yuhuangding East Road, Zhifu, Yantai, Shandong 264000, P.R. China

E-mail: ninebaby1006@126.com

${ }^{*}$ Contributed equally

Key words: pancreatic cancer, miR-29b-3p, methylation, invasion, metastasis, angiogenesis

\section{Introduction}

Pancreatic cancer is a highly malignant tumor of the digestive tract and the fourth leading cause of cancer-related death worldwide $(1,2)$, In recent years, its morbidity and mortality have exhibited an upward trend worldwide, due to difficulties in detection and lack of effective treatment, with a $\leq 5 \% 5$-year survival rate (2). In order to effectively diagnose, prevent, and treat this disease, further study of the molecular mechanism of pancreatic cancer is needed.

DNA methylation is a form of epigenetic modification and an important mechanism of gene expression regulation $(3,4)$. The occurrence and development of tumors are closely related to DNA methylation abnormalities, which are mainly manifested as a decrease in the overall genomic methylation level in tumor cells and an increase in the methylation level of the promoter region of specific genes (5-8). Previous studies have shown that methylation abnormalities in multiple promoter regions are closely related to the development of pancreatic cancer $(5,6)$.

As a member of the DNA methyltransferases (DNMTs), DNMT1 plays an important role in mediating gene expression and chromatin structure, by preserving existing DNA methylation during DNA replication (7). DNMT1 was found to be upregulated in various types of cancer, including pancreatic cancer (8). A study of pancreatic cancer demonstrated that lower expression of DNMT1 reversed the resistance to 5-azadeoxycytidine (9). In addition, siRNA targeting DNMT1 led to a reduction in cell viability and induced cell apoptosis in pancreatic cancer cells (10). Abnormal DNA methylation in tumors includes overall hypomethylation of the genome and hypermethylation of certain gene promoter regions. Abnormally elevated methylation of the promoter region may lead to transcriptional silencing of important regulatory genes such as cell cycle regulatory genes, tumor-suppressor genes, and apoptotic genes, resulting in decreased expression or loss of expression of related genes, and thereby promoting tumor formation (11). This hypermethylation is another mechanism leading to the inactivation of tumor-suppressor genes.

MicroRNAs (miRNAs) are a class of small non-coding RNAs with about 21-25 nucleotides, which can regulate the expression of post-transcriptional target genes $(12,13)$. A large number of researches also confirmed that miRNAs 
have the dual role of oncogene or tumor suppressor gene, and its expression changes are closely related to tumor formation (14-16). miR-29b-3p is a member of the miR-29 family and is closely related to the behavior of various tumors (17-21). In this study, we investigated the effects of the miR-29b-3p promoter methylation status on angiogenesis, invasion, and migration of pancreatic cancer cells. This study provides information concerning the role of the methylation of miR-29b in pancreatic cancer, and this may be a target for pancreatic cancer therapy.

\section{Materials and methods}

Patients and tissue collection. A total of 18 pairs of tissues from pancreatic cancer patients (mean age, 68.65 \pm 14.23 years ranging from 39 to $91 ; 8$ female patients and 10 male patients) were collected at the Yantai Yuhuangding Hospital of Qingdao University from March to November 2019. Patients who had received chemotherapy or radiotherapy were excluded in this study. The tissues were collected and transferred into liquid nitrogen and then were stored at $-80^{\circ} \mathrm{C}$. This study was approved by the Ethics Committee of the Yantai Yuhuangding Hospital of Qingdao University, and the ethics approval number is QDU-201902-3. All patients have provided written informed consent to participate in the study.

Antibodies, reagents, plasmids, miRNA, and siRNA. Antibodies against DNMT1 (dilution 1:1,000, cat. no. ab13537), DNMT2 (dilution 1:1,000, cat. no. ab272620), DNMT3a (dilution 1:1,000, cat. no. ab228691), DNMT3b (dilution 1:1,000, cat. no. ab122932), zonula occludens-1 (ZO-1) (dilution 1:1,000, cat. no. ab191143), occludin (dilution 1:1,000, cat. no. ab242202), claudin-5 (dilution 1:1,000, cat. no. ab15106), GAPDH (dilution 1:1,000, cat. no. ab9485) were from Abcam. HRP-labeled secondary antibodies (dilution 1:10,000, cat. no. sc-2370 or sc-2371) were from Santa Cruz Biotechnology, Inc. Fetal bovine serum (FBS, Gibco; Thermo Fisher Scientific, Inc.), Dulbecco's modified Eagle's medium (DMEM, Sigma; Merck KGaA, cat. no. 5796), Lipofectamine 3000 (Invitrogen; Thermo Fisher Scientific, Inc.), and NuPAGE 4-12\% Bis-Tris Gels were purchased from Thermo Fisher Scientific, Inc. Hydroquinone and sodium bisulfite were obtained from Sigma-Aldrich (Merck KGaA). Wizard DNA purification resin was obtained from Promega Corp. The CpGenome DNA Modification Kit was purchased from Intergen. The Vector and pcDNA3.1-DNMT1 were all designed and purchased from Invitrogen; Thermo Fisher Scientific, Inc. miR-29b-3p mimic, negative control mimics and all siRNA oligonucleotides were synthesized by GenePharma. All mimics and plasmids were transfected into cells using Lipofectamine 3000 (Invitrogen; Thermo Fisher Scientific, Inc). At 48-h post transfection, the transfected cells were collected for the next analysis.

Cell culture. Normal human pancreatic duct epithelial cells (HPDE6-C7) and 5 pancreatic cancer cell lines (BxPC3, PANC1, CFPAC, Capan-2, and AsPC-3) were purchased from Clontech. HUVECs were obtained from the American Type Culture Collection (ATCC). All cells were maintained and propagated in DMEM with $10 \% \mathrm{FBS}$ and $1 \%$ penicillin streptomycin in $5 \% \mathrm{CO}_{2}$ at $37^{\circ} \mathrm{C}$.
Western blotting. Cytoplasmic and nuclear protein fractions were extracted with the NE-PER Reagent Kit (Pierce; Thermo Fisher Scientific, Inc.) according to the manufacturer's instructions. Cell or tumor tissue lysates were separated by NuPAGE 4-12\% Bis-Tris Gels, under $60 \mathrm{~V}$ electrophoresis for $30 \mathrm{~min}$, followed by $120 \mathrm{~V}$ electrophoresis for $120 \mathrm{~min}$. After electrophoresis, proteins were transferred to PVDF membranes (Millipore), under $300 \mathrm{~mA}$ for $30 \mathrm{~min}$. The membrane was then blocked with $5 \%$ defatted milk powder for $60 \mathrm{~min}$ at room temperature. Mouse anti-human antibodies against DNMT1, DNMT2, DNMT3a, DNMT3b, ZO-1, occludin, claudin-5 and GAPDH (all diluted at 1:1,000, Santa Cruz Biotechnology, Inc.) were added at $4^{\circ} \mathrm{C}$ room temperature incubation overnight. The membrane was then washed with phosphate-buffered solution Tween (PBST) for $30 \mathrm{~min}$, followed by incubation with horseradish peroxidase (HRP)-conjugated secondary antibody for $60 \mathrm{~min}$ (dilution, 1:5,000, Santa Cruz Biotechnology, Inc.). After the membrane was washed three times with PBST, chemiluminescence detection reagent was used to develop the film. Gel image system was used to analyzed the band density (Bio-Rad Laboratories, Inc.).

Methylation-specific PCR. Genomic DNA was treated with bisulfite, and all cytosines that were not methylated were converted to uracil, while methylated cytosines were unchanged. Subsequently, the primers were designed for PCR at both ends of the CPG island to purify the target product. Primer pairs for PCR amplification were purchased from Thermo Fisher Scientific, Inc. After TA cloning, each clone was selected for positive clone sequencing, and finally the sequence was compared with the original sequence, the methylation site and number were counted, and the degree of methylation was analyzed.

Transient expression of DNMT1 in Bxpc3 and Capan-2 cells. The empty plasmid vector pcDNA3.1 (Invitrogen; Thermo Fisher Scientific, Inc.) or the plasmid vector containing DNMT1 cDNA was transfected into Bxpc3 and Capan-2 cells using Lipofectamine 3000 for subsequent experiments.

Transient transfection for functional analysis of miR-29b-3p and DNMT1. The cells were seeded in 6-well plates at $1 \times 10^{5}$ cells/well followed by culturing for $24 \mathrm{~h}$ and then transfected with $30 \mathrm{nM}$ of the miR-29b-3p mimic and the negative control mimics (NC) (GenePharma) using Lipofectamine 3000. The miR-29b-3p mimic and negative control mimic sequences were designed and synthesized by Gene pharma. The DNMT1 gene was knocked down by DNMT1 interfering small RNA (siRNA) obtained from Generay and transfected into the Bxpc3 and Capan-2 cells by Lipofectamine 3000 . The siRNA target sequence was as follows: DNMT1, 5'-TGTTAAGCTGTCTCTTTCCAA-3' and negative control, 5'-TAGATACTATGAATTCGTCCA A-3'. Medium was replaced with fresh medium after transfection for $6 \mathrm{~h}$, and the cells were cultured for another $48 \mathrm{~h}$ before further analysis.

Quantitative real-time PCR $(q P C R)$. The total RNA was isolated from cells using TRIzol reagent (Thermo Fisher Scientific, Inc.) according to the manufacturer's instructions. RNA $(1 \mu \mathrm{g})$ 
was converted into cDNA using the RevertAid ${ }^{\mathrm{TM}}$ First Strand cDNA Synthesis Kit (Fermentas; Thermo Fisher Scientific, Inc.). After 10-fold dilution, $4 \mu 1$ of cDNA was subjected to PCR amplification using SYBR Premix Ex Taq ${ }^{\mathrm{TM}}$ II (Takara) according to the manufacturer's protocol in a StepOnePlus ${ }^{\mathrm{TM}}$ Real-Time PCR System (ABI; Thermo Fisher Scientific, Inc.). The following thermocycling conditions were used for qPCR: $95^{\circ} \mathrm{C}$ for $10 \mathrm{sec}$, then 40 cycles with $95^{\circ} \mathrm{C}$ for $5 \mathrm{sec}, 60^{\circ} \mathrm{C}$ for 34 sec. $\beta$-actin served as the internal control. The primer sequences were as follows: DNMT1 forward, 5'-CCTAGCCCC AGGATTACAAGG-3' and reverse, 5'-ACTCATCCGATT TGGCTCTTTC-3'; miR-29b-3p forward, 5'-ACACTCCAG CTGGGTAGCACCATTTGAAATCA-3', reverse, 5'-CTC AACTGGTGTCGTGGA-3' and reverse transcription, 5'-CTC AACTGGTGTCGTGGAGTCGGCAATTCAGTTGAGAAC ACTGA-3'; $\beta$-actin forward, 5'-TGTTCGTCATGGGTGTGA AC-3' and reverse, 5'-ATGGCATGGACTGTGGTCAT-3'; U6 forward, 5'-CTCGCTTCGGCAGCACA-3' and U6 reverse, 5'-AACGCTTCACGAATTTGCGT-3'. $\beta$-actin and U6 were used as internal references for measuring relative expression of DNMT1 and miR-29b-3p, respectively. The expressions of genes were quantified using the $2^{-\Delta \Delta \mathrm{Cq}}$ method (22).

Cell migration and invasion assays (Transwell). Uncoated or Matrigel-coated chambers Transwells (BD Biosciences) containing $8-\mu \mathrm{m}$ pores were used for the assays. Cells (200 $\mu \mathrm{l})\left(1 \times 10^{5}\right.$ cells $\left./ \mathrm{ml}\right)$ were seeded into the upper chamber in serum-free DMEM medium. A total of $600 \mu \mathrm{l}$ conditioned DMEM media from target cells containing 10\% FBS was added to the lower chamber. Cells were fixed in $100 \%$ methanol $72 \mathrm{~h}$ later and stained with a 1:5 dilution of Giemsa (Sigma-Aldrich; Merck KGaA) for $40 \mathrm{~min}$ at room temperature. Cells remaining on the upper side of the filter were removed with a cotton swab. The filters were then mounted onto slides and images were captured under a microscope (Wetzlar, Germany, cat. no. DMI 1, Leica) at x200 magnification. From these images, the number of migratory or invasive cells was counted.

In vitro angiogenesis experiment of target cells co-cultured with HUVECs. The target cells $\left(1 \times 10^{5}\right.$ cells $\left./ \mathrm{ml}\right)$ were inoculated in a cell culture flask at the same density. After $6 \mathrm{~h}$ of culture, the culture medium was discarded and replaced with DMEM. After further culturing for $8 \mathrm{~h}$, the culture solution was collected and centrifuged at 1,000 x g for $10 \mathrm{~min}$ to collect the cell supernatant culture solution. Then $50 \mu \mathrm{l}$ of Matrigel was added to each well of a 96-well plate and incubated for $1 \mathrm{~h}$ at $37^{\circ} \mathrm{C}$. HUVECs were then added to the upper layer of Matrigel at $5 \times 10^{3}$ cells per well, and then incubated with the collected tumor cell culture supernatant. After 12-18 h, the formation of blood vessel-like structures of the HUVECs was observed and photographed under a fluorescence microscope (Keyence, cat. no. BZ-9000).

Statistics. All the quantitative data are represented as mean \pm SEM of at least three independent experiments. The difference between two groups was evaluated with the 2-tailed Student's t-test. One-way ANOVA and Tukey post hoc test were used to evaluate differences of multiple comparisons. All statistical analyses were conducted using GraphPad Prism software (version 7; GraphPad Software, Inc.). Differences were considered significant at $\mathrm{P}<0.05$.

\section{Results}

The miR-29b-3p gene promoter region methylation levels are increased, the DNMT1 expression levels are increased, and miR-29b-3p expression levels are decreased in pancreatic cancer. We identified one $\mathrm{CpG}$-rich region for each genomic locus of the miR-29b-3p promoter using MethPrimer (http://www.urogene.org/cgi-bin/methprimer/methprimer.cgi) and designed primer sets to analyze the $\mathrm{CpG}$-rich regions. Based on a database comparison, we predicted that the methylation level of the miR-29b-3p gene promoter region $(-3,000 \mathrm{bp})$ is increased in pancreatic cancer tissues (Fig. S1).

In the present study, we detected expression of DNMTs in pancreatic cancer tissues and adjacent tissues by western blot analysis. It was found that the expression level of DNMT1 in pancreatic cancer tissues was markedly higher than that in the adjacent tissues (Fig. 1A). DNMT1 expression was significant downregulated in adjacent tissues compared with tumor tissues, and this was used for later experiments. qPCR was used to detect expression of miR-29b-3p in the pancreatic cancer tissues, and the methylation levels of promoter regions were detected by pyrosequencing in pancreatic cancer tissues and adjacent tissues. It was found that the expression level of miR-29b-3p was decreased and this was negatively correlated with the methylation level of the miR-29b-3p promoter. $\left(\mathrm{R}^{2}=0.2162,1 /\right.$ slope $\left.=37.96\right)($ Fig. 1B $)$.

Six pancreatic cancer cell lines: HPDE6-C, BxPC3, PANC1, CFPAC, Capan-2, and AsPC-1 were cultured, and qPCR was used to detect the miR-29b-3p expression levels. The expression level of miR-29b-3p in BxPC3 and Capan-2 was found to be significantly lower than that of the other cell lines $(\mathrm{P}<0.01)$ (Fig. 1C). The methylation level of the miR-29b-3p gene promoter in these six pancreatic cancer cells was detected by BSP sequencing. It was found that BxPC3 and Capan-2 had more methylation sites and higher methylation levels (Fig. 1D).

Interference with expression of DNMT1 in Bxpc3 and Capan-2 cells in order to detect angiogenesis, invasion, and migration of pancreatic cancer cells. siRNA was utilized to interfere with DNMT1 in Bxpc3 and Capan-2 cells. qPCR revealed that the expression level of the miR-29b-3p gene in the siRNA DNMT1 group was significantly increased $(\mathrm{P}<0.001$ and $\mathrm{P}<0.01)$ (Fig. 2A). Western blot analysis revealed that expression of DNMT1 was decreased in the DNMT1 siRNA-transfected Bxpc3 and Capan-2 cells relative to that in the NC transfected Bxpc3 and Capan-2 cells, indicating that the interference effect was obvious (Fig. 2B).

Transwell assay showed that the migration and invasion abilities of pancreatic cancer cells in the siRNA DNMT1 group in Bxpc3 and Capan-2 cells were weakened, and the difference was statistically significant $(\mathrm{P}<0.01)$ (Fig. $2 \mathrm{C})$. Co-culture with HUVECs revealed that the angiogenic ability of the HUVECs was markedly attenuated after siRNA interference of DNMT1 expression (Fig. 2D). Western blotting found that the expression levels of ZO-1 and occludin were increased, and claudin-5 expression was decreased in 
A

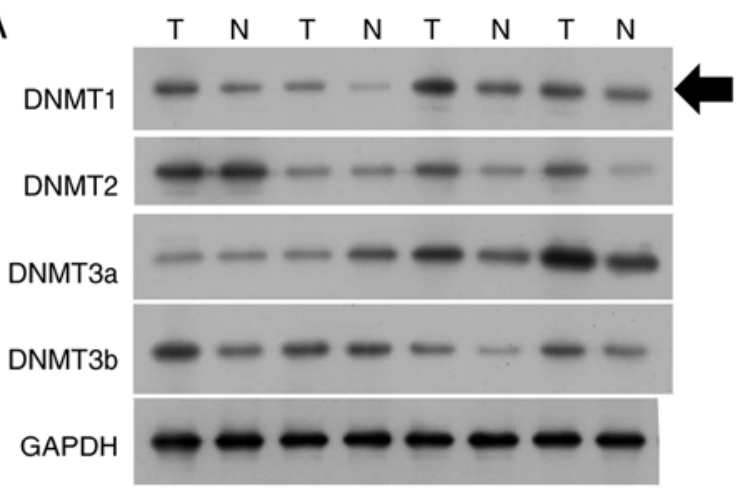

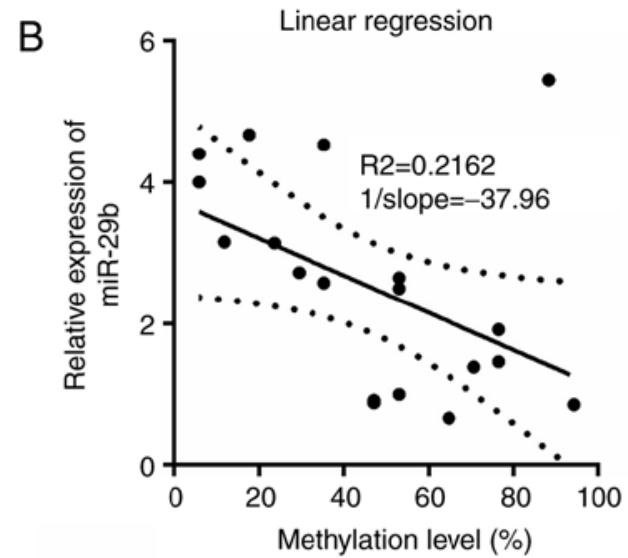

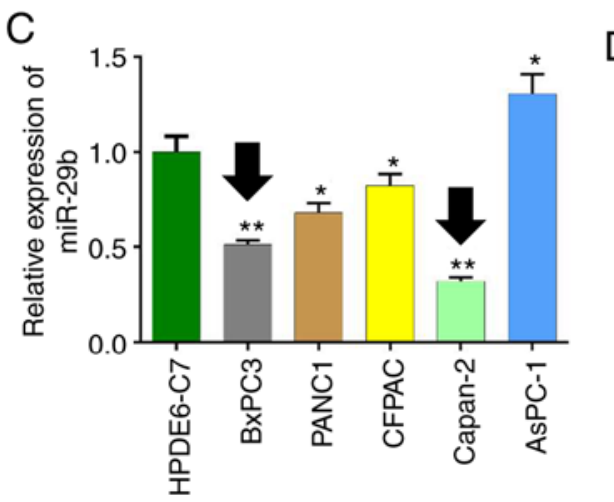

$\mathrm{D}_{\mathrm{H} P \mathrm{P}}$

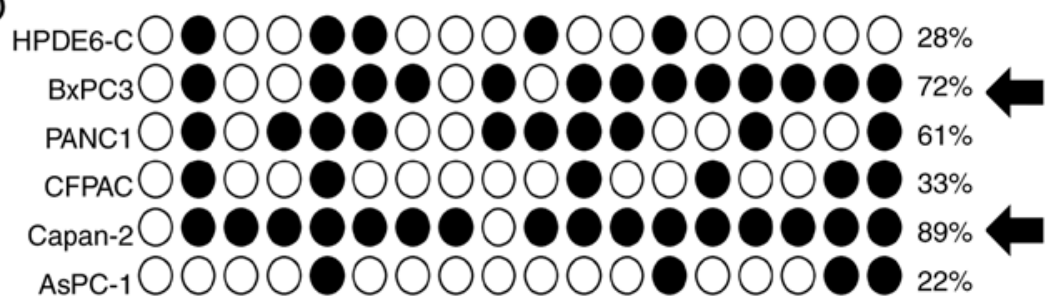

Figure 1. miR-29b-3p is negatively correlated with the methylation level of DNMT1 in pancreatic cancer. (A) Expression levels of DNMTs in pancreatic cancer tissues. (B) Correlation analysis of miR-29b-3p and the methylation level of DNMT1 in pancreatic cancer $\left(\mathrm{R}^{2}=0.2162\right)$. (C) The expression level of miR-29b-3p in normal human pancreatic duct epithelial cells (HPDE6-C7) and 5 pancreatic cancer cell lines (BxPC3, PANC1, CFPAC, Capan-2, and AsPC-3) ("P<0.05, ${ }^{* *} \mathrm{P}<0.01$ vs. HPDE6-C7 cells). (D) The methylation level of the miR-29b-3p gene promoter in pancreatic cancer cells. T, tumor tissues; N, non-tumor tissues; DNMTs, DNA methyltransferases.

the DNMT1 siRNA-transfected Bxpc3 and Capan-2 cells compared to that in the NC-transfected Bxpc3 and Capan-2 cells (Fig. 2E).

DNMT1-overexpressing Bxpc3 and Capan-2 cells were cultured, and miR-29b-3p mimic transfection was utilized in order to detect angiogenesis, invasion, and migration of pancreatic cancercells. Results of the qPCR found that DNMT1 expression was significantly increased $(\mathrm{P}<0.001), \mathrm{miR}-29 \mathrm{~b}-3 \mathrm{p}$ expression was significantly decreased $(\mathrm{P}<0.05, \mathrm{P}<0.001)$ in the DNMT1-overexpressed group compared with vector group; while the expression of DNMT1 was not significantly different in the DNMT1+miR-29b-3p group from that in the DNMT1 group, the expression of miR-29b-3p was significantly increased in DNMT1 and miR-29b-3p co-transfection group relative to that in DNMT1-overexpressed group $(\mathrm{P}<0.001)$ (Fig. 3A). Western blotting showed that DNMT1 expression in the DNMT1 group was higher than that in the NC group and the blank group, and the DNMT1+miR-29b-3p group had lower DNMT1 expression than the DNMT1 group (Fig. 3B). The results indicated that overexpression and interference were effective.

Transwell assay showed that the migration and invasive abilities of Bxpc3 and Capan-2 cells were significantly enhanced in the DNMT1 group vs. that in the vector group, while the enhancement of migration and invasion capacities mediated by DNMT1 overexpression were significantly weakened by miR-29b-3p in Capan-2 and BxPC3 cells $(\mathrm{P}<0.01)$ (Fig. 3C). Co-culture with HUVECs showed that the angiogenic ability of the HUVECs was enhanced in the DNMT1 group compared with that in the vector group, which also could be attenuated by miR-29b-3p addition in Capan-2 and BxPC3 cells (Fig. 3D). Western blotting analysis also discovered that ZO-1 and occludin expressions were markedly reduced, and claudin-5 expression was dramatically elevated in the DNMT1 overexpression group relative to that in the vector group, while the addition of miR-29b-3p then could prominently reverse the expression changes of ZO-1, occludin and claudin-5 in Bxpc3 and Capan-2 cells (Fig. 3E).

\section{Discussion}

DNA methylation in mammals means that methyl (-CH3) is covalently bound to the carbon atom of the cytosine (C) base of the DNA molecule under the catalysis of DNA methyltransferases (DNMTs) $(23,24)$. This usually occurs at the 5-position carbon atom of cytosine, forming 5-methylcytosine $(5 \mathrm{Mc})$, which is an epigenetic covalent modification process and the main way to inhibit gene expression and loss of function $(24,25)$.

Promoter methylation is involved in the early stage of cancer, and the degree of methylation increases with the increase in structural anomalies (26). Abnormal methylation of specific genes can be used as an indicator to judge the 

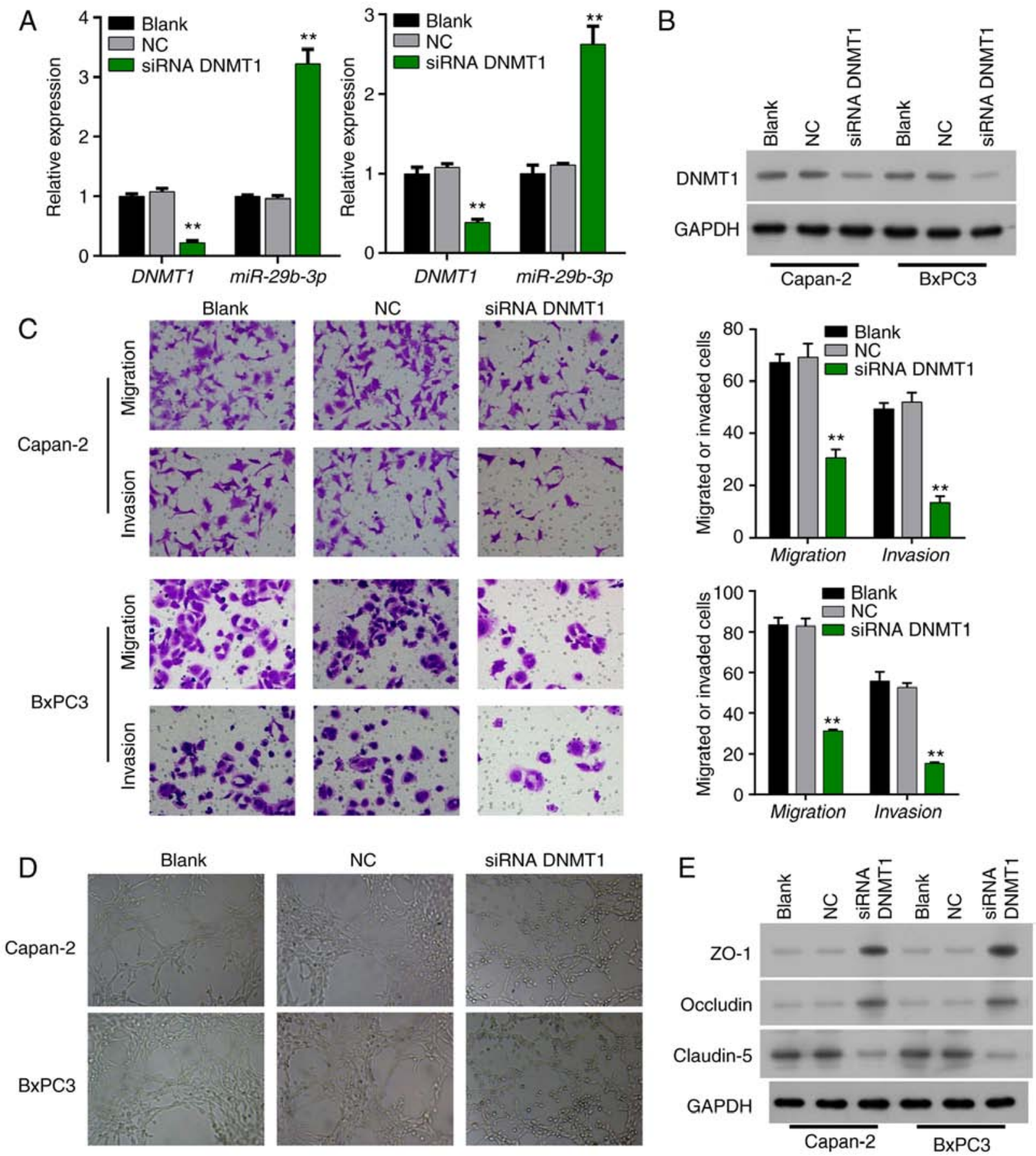

Figure 2. Knockdown of DNMT1 suppresses the methylation of miR-29b-3p and affects Capan-2 and BxPC3 cell migration and invasion. (A) Expression level of miR-29b-3p gene in cells transfected with DNMT1 siRNA. (B) Expression of DNMT1 in cells transfected with DNMT1 siRNA. (C) Migration and invasion of pancreatic cancer cells transfected with DNMT1 siRNA. (D) Angiogenic ability of HUVECs after siRNA interference of DNMT1. (E) Expression of ZO-1, claudin-5, and occludin in cells transfected with DNMT1 siRNA. ${ }^{* *} \mathrm{P}<0.01$, vs. NC group. $\mathrm{n}=3$. HUVECs, human umbilical vein endothelial cells; DNMT1, DNA methyltransferase 1; ZO-1, zonula occludens-1.

progression of pancreatic tumors (27). Numerous studies have shown that multiple gene methylation abnormalities are often detected in pancreatic cancer (28-31). It was also found that in precancerous lesions of pancreatic cancer, the methylation of the NPTX2 promoter increases with the degree of abnormal proliferation, suggesting that NPTX2 promoter regional hypermethylation is associated with early tumorigenesis in pancreatic cancer $(32,33)$. One study also found that pENK is highly methylated in pancreatic cancer tissue samples and pancreatic juice in pancreatic cancer patients, and its methylation to some extent promotes the formation of pancreatic cancer (34). In the present study, we found that the methylation of the miR-29 promoter was involved with malignant activities of pancreatic cancer cell lines. Overexpression of DNMT1 resulted in lower expression of miR-29, which led to cell migration, invasion, and angiogenesis.

MicroRNAs (miRNAs) are a family of non-coding RNAs that are very conservative and are approximately 15 to $25 \mathrm{nt}$ in length. In tumor research, according to the target gene of its downstream action, there are two major types of 


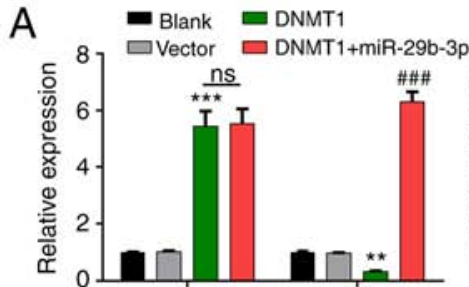

DNMT1 miR-29b-3p

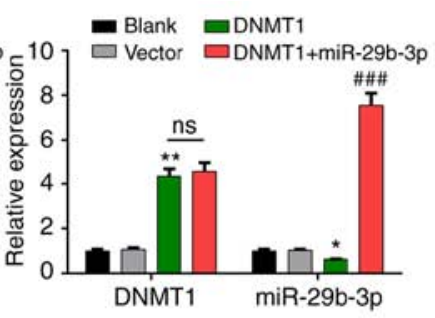

C

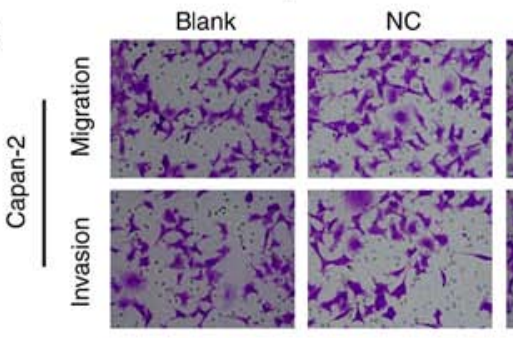

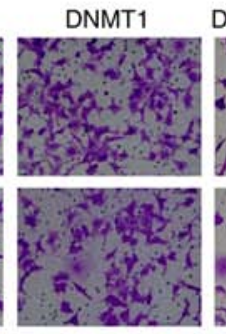

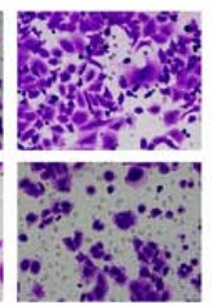

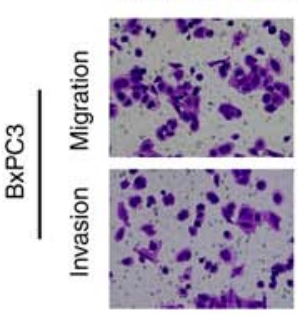

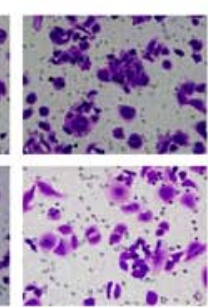

HUVEC

D
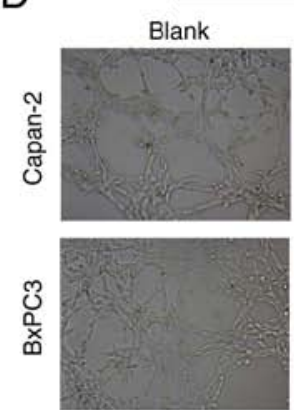

B
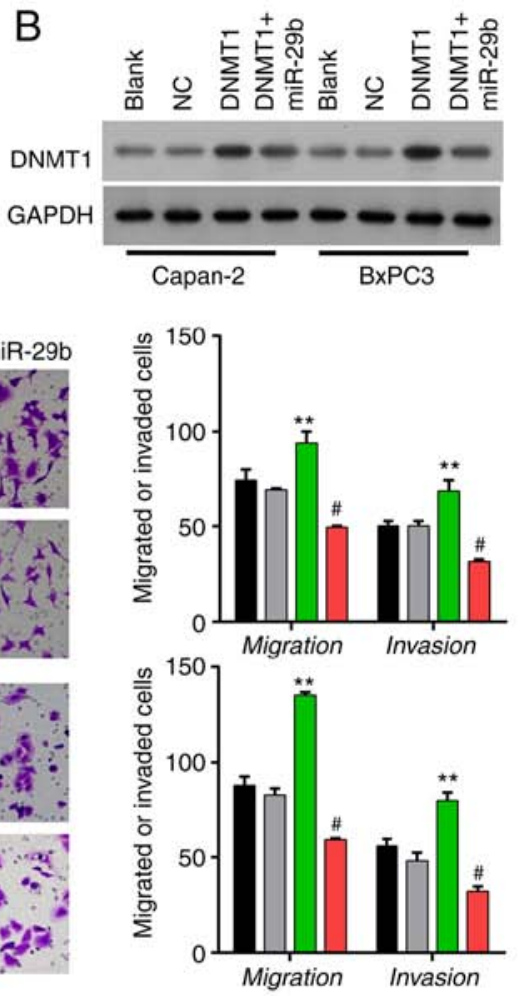

$\mathrm{E}$
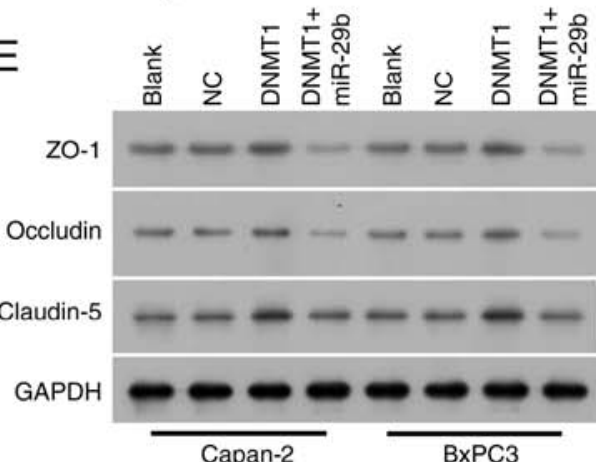

Figure 3. Methylation of the miR-29b-3p promoter contributes to angiogenesis, invasion, and migration in pancreatic cancer. (A) Expression of DNMT1 and miR-29b-3p in cells transfected with the DNMT1 expression plasmid and co-transfected with the DNMT1 expression plasmid and miR-29b-3p mimics in BxPC3 and Capan-2 cells. (B) Expression of DNMT1 in cells transfected with the DNMT1 expression plasmid and co-transfected with the DNMT1 expression plasmid and miR-29b-3p mimics. (C) The migration and invasive ability of pancreatic cancer cells in cells transfected with the DNMT1 expression plasmid and co-transfected with the DNMT1 expression plasmid and miR-29b-3p mimics. (D) The angiogenic ability of HUVECs co-cultured with pancreatic cancer cells transfected with the DNMT1 expression plasmid and co-transfected with the DNMT1 expression plasmid and miR-29b-3p mimics. (E) Expression of ZO-1, claudin-5, and occludin in cells transfected with the DNMT1 expression plasmid and co-transfected with the DNMT1 expression plasmid and miR-29b-3p mimics. HUVECs, human umbilical vein endothelial cells; DNMT1, DNA methyltransferase $1 ; \mathrm{ZO}-1$, zonula occludens-1. ${ }^{*} \mathrm{P}<0.05,{ }^{* *} \mathrm{P}<0.01,{ }^{* * *} \mathrm{P}<0.001$ vs. the Vector group; ${ }^{\#} \mathrm{P}<0.05,{ }^{\# \#} \mathrm{P}<0.001$ vs. the DNMT1 group; ns, not significant.

mircoRNAs, which are similar to the properties of oncogenes or tumor-suppressor genes (35). DNA aberrant methylation causes epigenetic silencing of some microRNAs and plays an important role in tumorigenesis and development (36). The human microRNA-29 (miRNA-29, miR-29) family is a group of small RNAs with the same seed sequence 'AGCACCA', including miR-29a, miR-29b, and miR-29c. There are miR-29 expression disorders in various tumor tissues, which are involved in expression of genes involved in tumor cell metabolism, proliferation, differentiation, and apoptosis through post-transcriptional regulation, and have the dual role of oncogene or tumor-suppressor gene (37). miR-29b-3p is a member of the miR-29 family and is involved in the development of pancreatic cancer $(38,39)$, colorectal cancer $(40)$, lung cancer (41), bladder cancer (19), and multiple myeloma (42). The relationship between miR-29b-3p promoter methylation and pancreatic cancer has not yet been reported. In the present study, we investigated the methylation of the miR-29b-3p promoter in pancreatic cancer and its expression level, and explored the effect of miR-29b-3p promoter methylation on angiogenesis, invasion, and migration of pancreatic cancer, thus providing a new theoretical basis for the treatment of pancreatic cancer.

It was found that the methylation level of the miR-29b-3p promoter region in pancreatic cancer tissues was significantly higher than that in adjacent tissues. In addition, the expression level of miR-29b-3p was significantly decreased, which was negatively correlated with the methylation 
level of its promoter. CpG methyltransferases (DNMTs) play a key role in DNA methylation, including DNMT1, DNMT2 DNMT3a, and DNMT3b. DNMT1 is the most important catalytic enzyme in the DNMT family. DNMT1 is associated with abnormal methylation of DNA and both are closely related to the occurrence and development of tumors $(43,44)$. The expression level of the DNMT1 protein in pancreatic cancer tissues was higher than that in adjacent tissues, suggesting that DNMT1 promotes promoter region methylation of the miR-29b-3p gene. siRNA was used to interfere with DNMT1 in Bxpc3 and Capan-2 cell lines, and expression of miR-29b-3p was significantly increased. We cultured DNMT1-overexpressing Bxpc3 and Capan-2, and expression of miR-29b-3p was significantly decreased. The above experiments proved that the methylation degree of the miR-29b-3p gene in pancreatic cancer leads to a change in its gene expression level, and the hypermethylation of the miR-29b-3p gene leads to its low expression.

Angiogenesis is the budding and subsequent stabilization of existing vascular wall cells (45). In 1973, Folkman first discovered that tumor cells induce angiogenesis and rapid growth, and since then, more and more attention has been paid to solid tumor angiogenesis (46). The vascular endothelial growth factor (VEGF), insulin-like growth factor 1 (IGF1), and other factors can play a role in promoting tumor angiogenesis, which is the basis of malignant tumor growth and metastasis (47-49). Zhang et al (50) found that exogenous low expression of miR-29a/c can increase expression and release of VEGF in gastric cancer cells, and promote the growth of vascular endothelial cells. Melo and Kalluri (51) found that miR-29b can inhibit the signaling molecules involved in angiogenesis and the extracellular matrix, such as VEGF, MMP9, ANGPTL4, and lysyloxidase (LOX), thereby inhibiting tumor angiogenesis and metastasis. This study investigated the role of miR-29b-3p in angiogenesis in pancreatic cancer cells, and found that miR-29b-3p inhibits angiogenesis and pancreatic cancer cell migration and invasion, and after inhibition of miR-29b-3p, the migration and invasive ability of pancreatic cancer cells increased. In this study, we aimed to investigate the role of DNMT1 and miR-29b-3p in pancreatic cancer, on cell migration, invasion and angiogenesis. However, the effect of DNMT1/miR-29b-3p on cell apoptosis and cycle was not investigated in the present study. Based on previous research, DNMT1 siRNA induces a significant cell viability decrease, leads to a G2-phase block and cell apoptosis in pancreatic cancer $(10,52)$, indicating that this axis may promote cell survival. Further study will focus on this aspect.

In conclusion, methylation of the miR-29b-3p promoter contributes to angiogenesis, invasion, and migration in pancreatic cancer. Its molecular mechanisms of regulating tumorigenesis and development need to be further studied.

\section{Acknowledgements}

Not applicable.

\section{Funding}

No funding was received.

\section{Availability of data and materials}

The datasets used and/or analyzed during the present study are available from the corresponding author on reasonable request.

\section{Authors' contributions}

LW designed the experiments. LW and NM performed the experiments, collected the data and analyzed the data. LW drafted the manuscript, and NQ validated the data analysis and revised the manuscript. All authors read and approved the manuscript and agree to be accountable for all aspects of the research in ensuring that the accuracy or integrity of any part of the work are appropriately investigated and resolved.

\section{Ethics approval and consent to participate}

This study was approved by the Ethics Committee of Yantai Yuhuangding Hospital of Qingdao University, and the ethics approval number is QDU-201902-3. Written informed consent was obtained from each participant.

\section{Patient consent for publication}

Not applicable.

\section{Competing interests}

The authors declare that they have no competing interests.

\section{References}

1. Zhu H, Li T, Du Y and Li M: Pancreatic cancer: Challenges and opportunities. BMC Med 16: 214, 2018.

2. Bray F, Ferlay J, Soerjomataram I, Siegel RL, Torre LA and Jemal A: Global cancer statistics 2018: GLOBOCAN estimates of incidence and mortality worldwide for 36 cancers in 185 countries. CA Cancer J Clin 68: 394-424, 2018.

3. Bogdanović $\mathrm{O}$ and Lister R: DNA methylation and the preservation of cell identity. Curr Opin Genet Dev 46: 9-14, 2017.

4. Zhang MW, Fujiwara K, Che X, Zheng S and Zheng L: DNA methylation in the tumor microenvironment. J Zhejiang Univ Sci B 18: 365-372, 2017.

5. Matsubayashi H, Canto M, Sato N, Klein A, Abe T, Yamashita K, Yeo CJ, Kalloo A, Hruban R and Goggins M: DNA methylation alterations in the pancreatic juice of patients with suspected pancreatic disease. Cancer Res 66: 1208-1217, 2006.

6. Pizzi S, Azzoni C, Bottarelli L, Campanini N, D'Adda T, Pasquali C, Rossi G, Rindi G and Bordi C: RASSF1A promoter methylation and 3p21.3 loss of heterozygosity are features of foregut, but not midgut and hindgut, malignant endocrine tumours. J Pathol 206: 409-416, 2005.

7. Bird A: DNA methylation patterns and epigenetic memory. Genes Dev 16: 6-21, 2002.

8. Hong L, Sun G, Peng L, Tu Y, Wan Z, Xiong H, Li Y and Xiao W: The interaction between miR-148a and DNMT1 suppresses cell migration and invasion by reactivating tumor suppressor genes in pancreatic cancer. Oncol Rep 40: 2916-2925, 2018.

9. Li A, Omura N, Hong SM and Goggins M: Pancreatic cancer DNMT1 expression and sensitivity to DNMT1 inhibitors. Cancer Biol Ther 9: 321-329, 2010.

10. Xu M, Gao J, Du YQ, Gao DJ, Zhang YQ, Li ZS, Zhang YL, Gong YF and Xu P: Reduction of pancreatic cancer cell viability and induction of apoptosis mediated by siRNA targeting DNMT1 through suppression of total DNA methyltransferase activity. Mol Med Rep 3: 699-704, 2010. 
11. Ikegami K, Ohgane J, Tanaka S, Yagi S and Shiota K: Interplay between DNA methylation, histone modification and chromatin remodeling in stem cells and during development. Int J Dev Biol 53: 203-214, 2009.

12. Cui J, Zhou B, Ross SA and Zempleni J: Nutrition, microRNAs, and human health. Adv Nutr 8: 105-112, 2017.

13. Lu TX and Rothenberg ME: MicroRNA. J Allergy Clin Immunol 141: 1202-1207, 2018.

14. Zhao J, Dong X, Liu QC and Lu Q: Expression of plasma miR-106a in epithelial ovarian cancer and its diagnostic and prognostic significance. Eur J Gynaecol Oncol 39: 769-772, 2018.

15. Ganju A, Khan S, Hafeez BB, Behrman SW, Yallapu MM, Chauhan SC and Jaggi M: miRNA nanotherapeutics for cancer. Drug Discov Today 22: 424-432, 2017.

16. Qadir MI and Faheem A: miRNA: A diagnostic and therapeutic tool for pancreatic cancer. Crit Rev Eukaryot Gene Expr 27: 197-204, 2017.

17. Li X, Xiao J, Fan Y, Yang K, Li K, Wang X, Lu Y and Zhou Y: miR-29 family regulates the puberty onset mediated by a novel Gnrh1 transcription factor TBX21. J Endocrinol 242: 185-197, 2019.

18. Ding D, Li C, Zhao T, Li D, Yang L and Zhang B: IncRNA H19/miR-29b-3p/PGRN axis promoted epithelial-mesenchymal transition of colorectal cancer cells by acting on wnt signaling. Mol Cells 41: 423-435, 2018.

19. Lv M, Zhong Z, Huang M, Tian Q, Jiang R and Chen J: lncRNA H19 regulates epithelial-mesenchymal transition and metastasis of bladder cancer by miR-29b-3p as competing endogenous RNA Biochim Biophys Acta Mol Cell Res 1864: 1887-1899, 2017.

20. Worst TS, Previti C, Nitschke K, Diessl N, Gross JC, Hoffmann L, Frey L, Thomas V, Kahlert C, Bieback K, et al: miR-10a-5p and miR-29b-3p as extracellular vesicle-associated prostate cancer detection markers. Cancers (Basel) 12: 43, 2019.

21. Zhang B, Shetti D, Fan C and Wei K: miR-29b-3p promotes progression of MDA-MB-231 triple-negative breast cancer cells through downregulating TRAF3. Biol Res 52: 38, 2019.

22. Feng L and Lou J: DNA methylation analysis. Methods Mol Biol 1894: 181-227, 2019.

23. Kasai $\mathrm{H}$ and Kawai K: DNA methylation at the C-5 position of cytosine by methyl radicals: A possible role for epigenetic change during carcinogenesis by environmental agents. Chem Res Toxicol 22: 984-989, 2009.

24. Przybilla J, Hopp L, Lübbert M, Loeffler M and Galle J: Targeting DNA hypermethylation: Computational modeling of DNA demethylation treatment of acute myeloid leukemia. Epigenetics 12: 886-896, 2017.

25. Kotandeniya D, Seiler CL, Fernandez J, Pujari SS, Curwick L, Murphy K, Wickramaratne S, Yan S, Murphy D, Sham YY and Tretyakova NY: Can 5-methylcytosine analogues with extended alkyl side chains guide DNA methylation? Chem Commun (Camb) 54: 1061-1064, 2018.

26. Yamashita K, Hosoda K, Nishizawa N, Katoh H and Watanabe M: Epigenetic biomarkers of promoter DNA methylation in the new era of cancer treatment. Cancer Sci 109: 3695-3706, 2018.

27. Van Tongelen A, Loriot A and De Smet C: Oncogenic roles of DNA hypomethylation through the activation of cancer-germline genes. Cancer Lett 396: 130-137, 2017.

28. Brancaccio M, Natale F, Falco G and Angrisano T: Cell-free DNA methylation: The new frontiers of pancreatic cancer biomarkers' discovery. Genes (Basel) 11: 14, 2019.

29. Mishra NK and Guda C: Genome-wide DNA methylation analysis reveals molecular subtypes of pancreatic cancer. Oncotarget 8: 28990-29012, 2017.

30. Natale F, Vivo M, Falco G and Angrisano T: Deciphering DNA methylation signatures of pancreatic cancer and pancreatitis. Clin Epigenetics 11: 132, 2019.

31. Zhou H, Zhu Y, Wei F, Shao Y, Pan J, Wang G, Xu K and Cheng Y: Significance of MUC2 gene methylation detection in pancreatic cancer diagnosis. Pancreatology 19: 1049-1053, 2019.

32. Park JK, Ji KR, Kim YT and Yong BY: Early diagnosis and aberrant methylation of NPTX2 gene in pancreatic cancer. Cancer Res 68: 5514, 2008.
33. Ling Z, Gao J, Li Z and Gong Y: Neuronal pentraxin II (NPTX2) is frequently down-regulated by promoter hypermethylation in pancreatic cancers. Dig Dis Sci 57: 2608-2614, 2012.

34. Singh N, Rashid S, Rashid S, Dash NR, Gupta S and Saraya A: Clinical significance of promoter methylation status of tumor suppressor genes in circulating DNA of pancreatic cancer patients. J Cancer Res Clin Oncol 146: 897-907, 2020.

35. Lujambio A and Lowe SW: The microcosmos of cancer. Nature 482: 347-355, 2012.

36. Moutinho $\mathrm{C}$ and Esteller M: MicroRNAs and Epigenetics. Adv Cancer Res 135: 189-220, 2017.

37. Jiang H, Zhang G, Wu JH and Jiang CP: Diverse roles of miR-29 in cancer (Review). Oncol Rep 31: 1509-1516, 2014.

38. Zhao X, Liu Y, Li Z, Zheng S, Wang Z, Li W, Bi Z, Li L, Jiang Y, Luo $Y$, et al: Linc00511 acts as a competing endogenous RNA to regulate VEGFA expression through sponging hsa-miR-29b-3p in pancreatic ductal adenocarcinoma. J Cell Mol Med 22: 655-667, 2018.

39. Sun Y, Wang P, Yang W, Shan Y, Zhang Q and Wu H: The role of lncRNA MSC-AS1/miR-29b-3p axis-mediated CDK14 modulation in pancreatic cancer proliferation and Gemcitabine-induced apoptosis. Cancer Biol Ther 20: 729-739, 2019.

40. Osawa T, Takeuchi A, Kojima T, Shinohara N, Eto M and Nishiyama H: Overview of current and future systemic therapy for metastatic renal cell carcinoma. Jpn J Clin Oncol 49: 395-403, 2019.

41. Li L, Liu Z, Jiang YY, Shen WX, Peng YP and Qiu YH: Acetylcholine suppresses microglial inflammatory response via $\alpha 7 \mathrm{nAChR}$ to protect hippocampal neurons. J Integr Neurosci 18: 51-56, 2019.

42. Liu D, Wang J and Liu M: Long noncoding RNA TUG1 promotes proliferation and inhibits apoptosis in multiple myeloma by inhibiting miR-29b-3p. Biosci Rep 39: BSR20182489, 2019.

43. Kim DH, Kim HM, Huong PTT, Han HJ, Hwang J, Cha-Molstad H, Lee KH, Ryoo IJ, Kim KE, Huh YH, et al: Enhanced anticancer effects of a methylation inhibitor by inhibiting a novel DNMT1 target, CEP 131, in cervical cancer. BMB Rep 52: 342-347, 2019.

44. Somasundaram S, Forrest ME, Moinova H, Cohen A, Varadan V, LaFramboise T, Markowitz S and Khalil AM: The DNMT1-associated lincRNADACOR1 reprograms genome-wide DNA methylation in colon cancer. Clin Epigenetics 10: 127, 2018.

45. Risau W: Mechanisms of angiogenesis. Nature 386: 671-674, 1997.

46. Folkman J: Tumor angiogenesis. Adv Cancer Res 19: 331-358, 1974.

47. Tang X, Zhang Q, Shi S, Yen Y, Li X, Zhang Y, Zhou K and Le AD: Bisphosphonates suppress insulin-like growth factor 1-induced angiogenesis via the HIF-1alpha/VEGF signaling pathways in human breast cancer cells. Int J Cancer 126: 90-103, 2010.

48. Zhou Y, Li S, Li J, Wang D and Li Q: Effect of microRNA-135a on cell proliferation, migration, invasion, apoptosis and tumor angiogenesis through the IGF-1/PI3K/Akt signaling pathway in non-small cell lung cancer. Cell Physiol Biochem 42: 1431-1446, 2017.

49. Azuma K, Suzuki S, Ishii Y, Ueda Y, Fujikawa T, Morinaga K, Shimoyama K and Oda J: Tortuosity of the brachiocephalic artery complicated with arterial injury after tracheotomy: A case report. Signa Vitae 15: 77-78, 2019.

50. Zhang H, Bai M, Deng T, Liu R, Wang X, Qu Y, Duan J, Zhang L, Ning T, Ge S, et al: Cell-derived microvesicles mediate the delivery of miR-29a/c to suppress angiogenesis in gastric carcinoma. Cancer Lett 375: 331-339, 2016.

51. Melo SA and Kalluri R: miR-29b moulds the tumour microenvironment to repress metastasis. Nat Cell Biol 15: 139-140, 2013.

52. Thakar M, Hu Y, Morreale M, Lerner L, Ying Lin W, Sen R, Cai Y, Karunasena E, Thakar M, Saggi S, et al: A novel epigenetic modulating agent sensitizes pancreatic cells to a chemotherapy agent. PLoS One 13: e0199130, 2018.

This work is licensed under a Creative Commons Attribution-NonCommercial-NoDerivatives 4.0 International (CC BY-NC-ND 4.0) License. 